\title{
Le contrôle de l'étalement urbain au Québec : 25 ans de valse-hésitation
}

\author{
Claire Binet
}

Qu'ils soient lassés de la ville ou simplement à la recherche d'un ailleurs meilleur, les urbains se sont lancés dans une reconquête de la campagne et ce, depuis une trentaine d'années. Dans un pays où la tradition de planification n'existait guère, cette recolonisation comporte un prix élevé : coûts collectifs importants liés à l'étalement urbain, notamment au plan des infrastructures, des équipements et services, sans compter un gaspillage de terres agricoles à haut potentiel et des tensions sociales liées aux problèmes de cohabitation entre les usages traditionnels et les nouvelles fonctions.

Le constat est lourd et les mécanismes d'arbitrage et de gestion du territoire fonctionnent plutôt mal. C'est à se demander s'il y a un pilote dans l'avion! Avant de se retrouver avec une quasi faillite des finances publiques sur les bras ou une intifada sociale, il conviendrait d'essayer de comprendre pourquoi il en est ainsi et consentir à une réflexion collective quant aux moyens adéquats pour redresser la situation.

\section{La recolonisation de la campagne}

Dans le monde occidental, la forme d'occupation prédominante de l'espace est urbaine. Au Québec, plus de $80 \%$ de la population vit sur moins de $5 \%$ du territoire. Cet habitat est à la fois concentré et dispersé, formant un chapelet de villes entourées de grandes surfaces urbanisées plus ou moins continues, à faible ou même très faible densité.

Cette forme étalée d'occupation est récente, s'étant développée dans la foulée des changements économiques et démographiques qui ont suivi la deuxième guerre mondiale. Depuis plusieurs décennies, l'absence sur le territoire québécois de tradition en matière de planification de l'aménagement au niveau local, que ce soit en milieu urbain ou rural, a fait en sorte que les décisions ont généralement découlé d'initiatives privées ponctuelles. En outre, l'État s'est longtemps abstenu d'intervenir et aucun modèle ne pouvait contrecarrer l'influence de la recette des métropoles américaines. Celle-ci a été appliquée d'abord par les grandes villes, bientôt imitées par les petites villes et ensuite même par des villages. Tout le monde voulait accéder au modèle gagnant représenté par la modernité et l'abondance de l'Amérique d'après-guerre.

Au Québec, les citadins sont plutôt de fraîche souche, car $80 \%$ de la population était rurale en 1860. Pour beaucoup de gens, les liens familiaux avec le milieu rural sont demeurés importants, mais ces liens se sont graduellement dilués, se transformant en une certaine nostalgie de la campagne dans un désir de faire contrepoids aux aspects négatifs de la ville. La proche campagne devient alors un espace à conquérir, dans un nouveau type de colonisation qui s'inscrit en continuité avec la tradition des ancêtres qui ont façonné le territoire.

Cette recolonisation, qui est à la fois résidence (saisonnière ou permanente) et récréation (golf, sentiers de randonnée), mais aussi économie (industrie, tourisme) et environnement (sites d'enfouissement, dépôts de pneus usés), modifie substantiellement les structures rurales existantes.

Une telle mouvance imprime divers impacts sur la société québécoise et sur son territoire, dont : 
- une urbanisation désordonnée qui engendre une demande pour des infrastructures, équipements et services, lesquels entraînent des coûts sociaux élevés à être assumés par les divers paliers de gouvernement;

- un exode des villes-centres vers les terrains moins chers de la périphérie, créant un déséquilibre fiscal, les centres continuant d'assurer différents services accessibles aux non-résidents;

- la disparition d'importantes superficies de terres arables de qualité et la déstructuration d'espaces agricoles considérables (morcellement, spéculation), ce qui contribue à entraver le développement de l'agriculture.

À cause des particularités géographiques du Québec, c'est le plus souvent à même des espaces agricoles que s'effectue cette « recolonisation » de la campagne par les urbains. Conséquemment, la question des espaces agricoles occupera ici une place privilégiée.

\section{Une proche campagne très agricole}

Rappelons d'abord que, historiquement, les centres urbains se sont implantés près de terres agricoles assurant leur approvisionnement. Ainsi, 22 des 31 agglomérations québécoises de plus de 10000 habitants sont situées sur ou à proximité de terres agricoles à potentiel élevé. La problématique des conflits d'utilisation du sol reliés à une telle juxtaposition est au Québec exacerbée par des facteurs spécifiques à son agriculture.

Voyons-en quelques-uns. En premier lieu, les terres les plus aptes à la production sont surtout situées dans la vallée du Saint-Laurent, où vivent environ les trois quarts de la population québécoise, la moitié des terres de qualité supérieure étant concentrée dans la plaine de Montréal. En deuxième lieu, les conditions bioclimatiques font qu'à peine $2 \%$ du territoire du Québec sont constitués de terres cultivables, tous potentiels confondus. En troisième lieu, la valeur de la production agricole du Québec est regroupée dans un rayon d'environ une heure de route des six agglomérations de plus de 100000 habitants. En quatrième lieu, comme les productions animales représentent les trois quarts de la valeur de l'agriculture québécoise, des problèmes de cohabitation sont fréquents, les rési-

dences et les activités récréotouristiques avoisinant l'agriculture. Il ne saurait être ici question d'évacuer la problématique complexe des rapports entre, d'une part, une agriculture moderne et productive et, d'autre part, la cohabitation des activités et la protection de l'environnement car cela nécessiterait une analyse distincte.

\section{En principe, des outils de gestion du territoire permettent d'arbitrer les divers intérêts en cause, mais les résultats sont loin d'être convaincants.}

Par ailleurs, ajoutons que l'économie rurale est tributaire du secteur primaire. Comparativement à d'autres activités primaires plus vulnérables aux fluctuations des marchés mondiaux (secteurs des mines et des forêts), l'agriculture constitue un élément stabilisateur du milieu rural ${ }^{1}$. Cette activité concourt à nourrir la population à un coût du panier d'épicerie parmi les plus bas au monde, mais elle participe également pour moitié à la transformation bioalimentaire du Québec. Fortement concentrées à Montréal et dans sa périphérie, ces usines se positionnent dans le peloton de tête des activités manufacturières au Québec. Pris globalement, le secteur bioalimentaire emploie presque une personne sur huit au Québec; dans la région métropolitaine de Montréal, au moins $10 \%$ des emplois y sont reliés ${ }^{2}$.

La réunion de ces divers ingrédients contribue à mettre la table pour une recolonisation litigieuse, où des objectifs économiques et sociaux divergents s'opposent. En principe, des outils de gestion du territoire permettent d'arbitrer les divers intérêts en cause, mais les résultats sont loin d'être convaincants. Nous allons maintenant porter un regard critique sur ces outils d'intervention que sont le zonage agricole et les schémas d'aménagement.

\section{Comme un pendule qui oscille}

La Loi sur la protection du territoire agricole (LPTA) a été adoptée en 1978 dans un contexte d'urgence. D'effet immédiat, elle a été comme un coup de massue pour les acteurs du développement immobilier et pour le monde municipal. Le plus souvent appliquée de manière sévère au cours des premières années, cette loi est vite devenue une cause majeure 
d'irritants. Dès le début, les élus municipaux ont joué les Ponce Pilate et, encore aujourd'hui, beaucoup de municipalités appuient massivement la plupart des demandes à la Commission de protection du territoire agricole (CPTA), laissant à cette dernière la responsabilité d'opposer un refus.

Une modification législative en 1985 prévoyait une révision de la zone agricole pour arrimer les «zones blanches » aux schémas d'aménagement alors en élaboration. Cependant, l'élection d'un nouveau gouvernement, qui avait fait campagne sur la promesse d'éliminer les irritants de cette loi, a transformé l'ajustement prévu en une renégociation complète de la zone agricole. Cette révision s'est déroulée entre 1986 et 1992 dans un climat de contestation et de scandale, l'Union des producteurs agricoles (UPA) et divers organismes (dont la Communauté urbaine de Montréal) accusant la CPTA de trop concéder aux municipalités. Au total, plus de 200000 ha ont été exclus. Dans la grande région de Montréal, les exclusions ont visé une superficie équivalente à celle de l'île de Montréal, soit 48000 ha, essentiellement des sols à haut potentiel.

Entre-temps, diverses nominations à la CPTA et des modifications législatives en 1989 ont facilité l'obtention d'autorisations. Entre 1993 et 1995, le taux moyen d'autorisation des demandes était de $70 \%$ dans l'ensemble du Québec. Pour les demandes à des fins résidentielles, ce taux était d'environ $40 \%$, mais il était généralement au-dessus de $90 \%$ pour les projets d'utilité publique ${ }^{3}$. En 1993, la CPTA a fait l'objet d'un examen par le Vérificateur général du Québec qui l'a sévèrement critiquée pour son laxisme de même que pour son manque de vision et de cohérence. L'année suivante, un autre changement de gouvernement a entraîné un certain réalignement, visant un resserrement de la protection du territoire agricole.

Malgré que la révision de la zone agricole ait accordé des superficies suffisantes pour absorber le développement urbain des 30 ou 40 prochaines années, les demandes ont continué à inonder la CPTA. Celle-ci a parfois accordé des exclusions à des MRC sans que les besoins d'espaces à urbaniser n'aient été démontrés auprès du ministère des Affaires municipales, chargé de coordonner la révision des schémas d'aménagement actuellement en cours. Le gouvernement a dû récemment y remédier par voie législative en ajustant les deux processus.
À la suite des nombreuses mesures de revitalisation mises en place après 1976, il est difficile d'évaluer l'impact du zonage agricole dans la relance de l'agriculture. Après une forte régression de 1956 à 1971, suivie d'une quasi stagnation jusqu'en 1996, les surfaces cultivées ont augmenté dans l'ensemble du Québec. En 2001, le niveau de 1971 a pu enfin être rejoint et dépassé, avec 1849938 hectares. Pour certaines régions, les progrès ont été modestes mais, en Montérégie, la hausse a été considérable.

Ce rattrapage notable des superficies en production en Montérégie a varié d'une MRC à l'autre, notamment en fonction de leur proximité de l'agglomération montréalaise : $57 \%$ entre 1971 et 2001 dans Les Maskoutains, $33 \%$ dans Le Bas-Richelieu, $10 \%$ dans Roussillon, 4,5\% dans Lajemmerais ${ }^{4}$. Pendant la période de révision de la zone agricole (1986-1991), plusieurs gains récents ont été annulés et de plus fortes exclusions ont été consenties dans les MRC de la frange urbaine (1\%-2\% dans Les Maskoutains et Le Bas-Richelieu, $10 \%$ dans Lajemmerais, $5 \%$ dans Roussillon) ${ }^{5}$. Après l'incertitude de la période de révision, la tendance générale est maintenant à la hausse dans l'ensemble de la Montérégie, surtout depuis 1996.

\section{Il est difficile d'évaluer l'impact du zonage agricole dans la relance de l'agriculture.}

Mentionnons que, au moment de l'entrée en vigueur de la LPTA, l'agriculture vivotait un peu au Québec et les espaces agricoles étaient fortement déstructurés dans plusieurs milieux, particulièrement dans la frange urbaine de la couronne sud montréalaise. La MRC de Lajemmerais était d'ailleurs fortement touchée ${ }^{6}$. Les friches occupaient des proportions notables de la zone agricole, notamment dans les MRC de Lajemmerais et Roussillon où plus du tiers des terres était en location. La reconquête agricole s'est d'abord effectuée sur des friches récentes, les plus anciennes impliquant d'importants coûts de remise en valeur. Depuis, le taux de location a baissé dans Roussillon, mais il a augmenté dans Lajemmerais.

L'importance du phénomène de la location contribue à fragiliser les activités agricoles $(29 \%$ des terres en culture de la région métropolitaine de Montréal sont 
louées) ${ }^{7}$ et témoigne d'une spéculation persistante. En outre, signalons que la location, souvent à court terme, ne favorise ni les investissements pour des améliorations du sol (drainage, chaulage) ni des plantations pour des productions à plus ou moins long terme (fraisiers, vignes, arbres fruitiers). En outre, dans plusieurs secteurs, les surfaces disponibles pour l'ensemencement se raréfient et certains agriculteurs ont entrepris de s'agrandir en défrichant leurs boisés de ferme, alors qu'il reste très peu de couvert forestier en Montérégie.

À l'intérieur des MRC, les municipalités jouxtant l'agglomération demeurent plus vulnérables. Ainsi, dans Lajemmerais, les taux de location des terres sont plus élevés à Boucherville, Sainte-Julie et Varennes. De plus, la ponction a été plus forte dans ces municipalités lors de la révision de la zone agricole et les superficies en friche y étaient plus importantes ${ }^{8}$. Dans l'ensemble de cette MRC, les espaces agricoles apparaissent fragilisés et ce, malgré une certaine vigueur qui pourrait n'être que temporaire.

\section{Dans les schémas d'aménagement de première génération, la plupart des MRC permettaient pratiquement tous les usages dans les affectations du sol en zone agricole.}

L'application plus ou moins rigoureuse de la LPTA, les mesures qui l'ont tout à tour affaiblie ou renforcée de même que la révision de la zone agricole ont fait en sorte que, globalement, la mise en œuvre de cette loi semble avoir peu contribué à contrôler l'étalement urbain sur les terres agricoles. Tout au plus a-t-elle pu le ralentir, le circonscrire, le séquencer. Elle a néanmoins permis de mettre un peu d'ordre dans des initiatives débraillées comme le développement en saute-mouton, qui mitait les espaces agricoles.

Par ailleurs, la catastrophe anticipée d'une pénurie de lots à bâtir n'a pas eu lieu; sur la rive sud de Montréal, le prix des terres agricoles a même augmenté plus vite que celui des terrains résidentiels ${ }^{9}$. De plus, la revitalisation des espaces agricoles périurbains a été entravée par des pressions urbaines persistantes. Plusieurs demandes d'exclusion sont actuellement en attente d'une décision de la CPTA.

\section{Comme des radeaux en dérive sur une mer urbaine étale}

Adoptée peu après la LPTA, la Loi sur l'aménagement et l'urbanisme (LAU) a été mise en œuvre par étape. Dès le début, les exigences reliées à l'élaboration des schémas d'aménagement ont dérouté les élus municipaux, habitués à une référence territoriale qui débordait peu les limites locales; de plus, la planification et l'arbitrage des «querelles de clocher » ne faisaient guère partie de la culture municipale d'alors.

Du côté gouvernemental, les orientations pour l'élaboration des schémas d'aménagement ont été publiées tardivement (1983), chaque ministère exposant ses préoccupations et projets. Par ailleurs, l'Orientation préférable d'aménagement pour la région de Montréal, publiée en 1978 et réaffirmée en 1983, préconisait de «construire la ville en ville» : consolidation du tissu urbain à l'intérieur du périmètre bâti et viabilisé, réaménagement prioritaire des territoires anciennement bâtis, amélioration de la qualité de vie sur l'île de Montréal. Elle allait heurter de front la culture dominante chez les décideurs, et pas seulement dans le milieu municipal.

Le contexte dans lequel s'amorçait l'élaboration des schémas d'aménagement contenait des ingrédients annonciateurs d'une démarche laborieuse et chaotique, ce qui fut le cas. D'une part, le consensus et la cohérence faisaient défaut au départ dans l'appareil gouvernemental. D'autre part, bon nombre d'élus municipaux étaient d'emblée contrariés et amers, estimant avoir été dépouillés de leurs pouvoirs sur une grande partie du territoire à la suite des récents décrets de zone agricole.

Dans les schémas d'aménagement de première génération, la plupart des MRC permettaient pratiquement tous les usages dans les affectations du sol en zone agricole, laissant à la CPTA la charge de trancher face aux demandes qui lui étaient soumises. Une analyse de quelques schémas d'aménagement de la région montréalaise a révélé que la préoccupation de planifier l'urbanisation était alors peu présente; celle de protéger le territoire agricole l'était encore moins ${ }^{10}$.

Il serait trop facile d'attribuer au changement de gouvernement survenu à la fin de 1985 les longs délais de mise en œuvre et le fait que les orientations 
gouvernementales aient été plus ou moins appliquées, surtout celle relative à l'Option préférable d'aménagement pour la région de Montréal. À une exception près, les schémas d'aménagement sont entrés en vigueur de quatre à sept ans après la publication des orientations gouvernementales. Il fallait ensuite entreprendre la confection des plans d'urbanisme des municipalités et l'adoption de leurs règlements d'urbanisme. On n'était pas sorti du bois! Dans beaucoup de cas, cette démarche a nécessité presque autant de temps que l'élaboration des schémas. Arrivé au terme du processus, la durée de validité des schémas était terminée et il fallait les réviser. Le ministère des Affaires municipales a perdu le contrôle des échéanciers, se montrant plus que compréhensif face aux multiples demandes d'extension de délai de MRC.

Par ailleurs, diverses politiques sectorielles structurantes pour l'urbanisation ont été mises en œuvre parallèlement à l'adoption des schémas d'aménagement. Mentionnons les programmes d'aide pour l'accès à la propriété, la levée du moratoire sur les autoroutes et les importantes mises de fond gouvernementales pour les infrastructures de traitement des eaux usées. Il faut aussi souligner le financement de divers équipements et services à la population (écoles, hôpitaux, bibliothèques, etc.) qui a facilité l'implantation de nouvelles populations en banlieue ou en périphérie des villages.

L'arbitrage politique au sein du gouvernement n'a pas réussi à coordonner les interventions sectorielles. Cette faiblesse a été amplifiée à partir de 1991 par l'abolition du comité ministériel chargé d'examiner les questions relatives à l'aménagement et au développement du territoire (COMPADRE). Depuis, même si les autres ministères fournissent des avis techniques, le ministre des Affaires municipales est seul à décider de la conformité d'un schéma d'aménagement aux orientations gouvernementales.

Constatant que le développement de la région montréalaise ne s'harmonisait guère, le gouvernement du Québec a créé en 1992 un groupe de travail. Déposé l'année suivante, le rapport Pichette préconisait un grand coup de barre qui a provoqué une levée de boucliers dans le monde municipal : abolition des MRC de la région montréalaise, planification régionale centralisée, mesures de péréquation et de responsabilisation face aux coûts d'urbanisation.

Aux prises avec une importante crise des finances publiques et cherchant à rationaliser les dépenses sur le territoire, le gouvernement a alors inclus un volet spécifique sur la gestion de l'urbanisation dans les orientations gouvernementales pour la révision des schémas d'aménagement publiées en 1994. L'élection d'un nouveau gouvernement plus tard cette année-là a débouché sur un resserrement de l'encadrement pour la gestion de l'urbanisation de même que des mesures pour la protection et la mise en valeur du territoire agricole ${ }^{11}$, ce qui a contrarié les ambitions de beaucoup de MRC.

\section{Beaucoup de MRC ne voient pas l'intérêt de se presser pour réviser leur schéma d'aménagement.}

Les premiers schémas révisés sont entrés en vigueur en 1998 et la progression demeure très lente : moins du tiers des MRC a un schéma révisé à la fin de 2003. Entre-temps, la population a encore changé de gouvernement. Considérant que la révision des schémas dure déjà depuis presque dix ans et que, en principe, ceux-ci ont une durée de vie de cinq ans, force est de constater que le mécanisme de la LAU est nettement paralysé. Encore une fois, le ministère des Affaires municipales est permissif face à l'échéancier, continuant plutôt avec stoïcisme sa démarche de sensibilisation pour une meilleure gestion de l'urbanisation.

Il est évident que le resserrement des orientations gouvernementales en matière de gestion de l'urbanisation et les modifications à la LPTA adoptées en 1996 et en 2001 sont les principales raisons expliquant ce dysfonctionnement. En effet, beaucoup de MRC ne voient pas l'intérêt de se presser pour réviser leur schéma d'aménagement, car des règles particulières de la LPTA s'appliquent aux schémas révisés, rendant les exclusions de la zone agricole plus difficiles. Précisons que la révision des schémas d'aménagement prend place dans un climat socio-politique tendu entre les municipalités et le monde agricole sur certaines questions : l'identification des usages non agricoles permis en zone agricole, de même que l'encadrement de la cohabitation de ces usages avec les activités agricoles. Cette polémique complexe sur la conformité de diverses positions par rapport aux orientations gouvernementales pose une question cruciale : sur la base de quels critères la conformité d'un schéma 
d'aménagement est-elle établie ? Étant donné que le ministre des Affaires municipales a le dernier mot à ce sujet, il est exposé à subir de fortes pressions de sa clientèle.

En désespoir de cause, le gouvernement a fait une autre tentative pour accélérer la révision des schémas en adoptant des mesures particulières en 2001, mais il semble que diverses astuces permettent aux MRC de les contourner en partie. On peut d'ailleurs se demander si la stratégie de nombreux élus municipaux ne serait pas de gagner du temps dans l'espoir que le récent changement de gouvernement ne vienne alléger les règles concernant la zone agricole. Dans ce cas, gagneront-ils leur pari?

\section{Pendant ce temps, sur le terrain...}

Il n'existe pas encore d'instrument accessible pour mesurer la portée concrète de l'application de la LPTA et de la LAU dans une municipalité ou une MRC, notamment par une vue d'ensemble des pressions sur la zone agricole. Cependant, notre étude effectuée en 1999 sur le territoire de la rive-sud de Montréal, et plus particulièrement sur celui de la MRC de Lajemmerais, a permis d'apporter un éclairage à ce propos ${ }^{12}$.

\section{Les faits démontrent que l'érosion des espaces en culture est plus vive au contact de surfaces urbanisées ou industrielles.}

Le décret de révision de 1990 a soustrait plus de 3100 hectares à la zone agricole de cette MRC, soit près de $10 \%$ de la superficie zonée en 1980 , le territoire demeurant zoné agricole à $71 \%$. En 2002, la CPTA a reçu des demandes d'exclusion couvrant quelque 500 hectares pour trois municipalités, dont 380 hectares pour Sainte-Julie seulement (qui en avait pourtant obtenu 365 avec la révision). Une telle situation découle du fait que, dans sa première version de schéma révisé adoptée en 1995, la MRC de Lajemmerais prenait le parti de concurrencer les nouveaux projets résidentiels du centre de l'agglomération et de favoriser l'expansion des aires industrielles existantes. Ce projet, plus menaçant pour la pérennité de la zone agricole que le schéma existant, allait directement à l'encontre des orientations gouvernementales.
D'interminables discussions ont mené à l'adoption d'un schéma révisé en 2003, mais l'approbation gouvernementale reste à venir.

Un des volets de cette étude comportait une analyse détaillée de 13 paires de secteurs d'environ 300 à 350 hectares chacun, totalisant près de 9000 hectares (le quart de la zone agricole). La moitié de ces secteurs était déstructurée et adjacente à des noyaux urbains ou à des espaces industriels; chacun d'eux était jumelé à un secteur agricole plus homogène, localisé à proximité.

L'exercice a consisté à cartographier en superposition, pour chacun des 26 secteurs délimités, les informations suivantes: l'évolution de l'utilisation du sol (couvrant une période de près de 20 ans), l'application globale du zonage agricole (évolution des limites de la zone agricole et autorisations de la CPTA) et les affectations du sol des schémas d'aménagement (en vigueur et projet de schéma révisé). Les faits démontrent que l'érosion des espaces en culture est plus vive au contact de surfaces urbanisées ou industrielles. Elle s'amplifie si la protection de la LPTA disparait (exclusions) ou diminue (autorisations). Il en de même si une affectation non agricole est attribuée par le schéma. Quand les deux effets se combinent, les surfaces cultivées se réduisent comme une peau de chagrin. Les secteurs agricoles homogènes ne sont pas à l'abri et sont également touchés par effet d'entraînement. En outre, de nouvelles friches apparaissent, ce qui pourrait être un indice de spéculation accrue, ces messages officiels des autorités pouvant être interprétés comme une invitation à passer à l'urbanisation.

Ces constats s'ajoutent à ceux présentés ci-dessus au sujet de l'évolution de la zone agricole et des superficies en production dans les municipalités composantes de Lajemmetais et dans les MRC de la rive-sud de Montréal. Il est clair que, peu importe l'échelle d'observation, l'urbanisation continue de consommer des terres agricoles de qualité. De plus, les pressions urbaines contribuent à déstructurer les espaces agricoles, même sur des superficies qui ne seront vraisemblablement jamais urbanisées, et à freiner la revitalisation de l'agriculture autour des centres urbains.

Le cas de la MRC de Lajemmerais n'est sans doute pas exceptionnel et pourrait être indicateur d'une tendance répandue à divers degrés, et pas uniquement dans cette région. La manifestation de ce phénomène 
pourrait être mise en lumière dans beaucoup de municipalités situées autour des principales agglomérations du Québec, dans le voisinage de villes de moindre importance, autour de centres de services et même de beaucoup de villages. Presque 25 ans après l'introduction de la LPTA et de la LAU dans le paysage municipal, les résistances demeurent encore très vives et l'étalement urbain sur les terres agricoles perdure.

\section{Des rentrées de taxes à tout prix}

Dans la logique municipale de gestion de l'espace, la recherche de revenus est une importante motivation des élus, ce qui est a priori fort légitime. Les taxes foncières représentent en effet l'essentiel du revenu des municipalités, surtout depuis la réforme de la fiscalité municipale de 1979 qui leur a enlevé l'accès à une portion de la taxe de vente perçue par le gouvernement québécois, laquelle pouvait jusqu'alors constituer une forme d'incitation à favoriser un développement économique diversifié.

La valeur foncière joue un rôle déterminant en matière de gestion de l'espace. Les théories d'économie spatiale (Christaller et autres) ont clairement démontré l'importance de la centralité et de la rente foncière dans la localisation des diverses activités sur un territoire. Étant donné la concurrence entre les différentes activités pour occuper les sites stratégiques reliés à cette centralité, celles générant le plus de rentabilité directe l'emportent, à moins que des règles externes (lois, règlements, normes) ne viennent modifier le jeu du libre marché. Dans de tels cas, des pressions et intérêts sectoriels se manifestent pour influencer cet encadrement ou y déroger, ce qui a été abondamment illustré dans les lignes précédentes.

Pour des élus municipaux désireux de voir s'accroître la richesse foncière et augmenter les rentrées de taxes, la construction de bâtiments est devenue la voie privilégiée. À cet égard, les urbains à la recherche d'un espace plus aéré et intimiste sont séduits par des terrains moins chers de même que des taxes foncières moins élevées. La vocation résidentielle est donc devenue «la vache à lait » des municipalités périurbaines qui sont en compétition pour attirer de nouveaux résidents de la classe moyenne qui abandonnent les villes-centres. Dans cette foulée, d'autres activités économiques migrent à la périphérie (com-

merces de grande surface, industries consommatrices d'espace).

\section{Il n'a encore jamais été démontré que le développement domiciliaire est rentable pour les municipalités.}

À moins d'être considérée pour d'autres apports à la communauté que la production, l'agriculture ne peut concurrencer les autres vocations qui, au prorata de la superficie, rapportent plus de taxes aux municipalités. Dans ce contexte, l'importante contribution de l'agriculture à l'économie québécoise est peu perceptible localement. Le fait que l'agriculture présente un des plus hauts niveaux de capitalisation par rapport à d'autres activités fragilise encore plus sa position sur l'échiquier municipal. Quand des édiles municipaux parlent de développement, cela exclut presque toujours l'agriculture. Cette perception demeure, même si elle est un secteur économique qui coûte très peu en termes de service. Soulignons que, malgré la valeur foncière en général plus basse des entreprises agricoles comparativement à celle d'autres types d'activité, elle constitue néanmoins la plus grande part de l'assiette foncière de la majorité des municipalités rurales.

Il n'a encore jamais été démontré que le développement domiciliaire est rentable pour les municipalités. En réalité, les nouvelles constructions résidentielles entraînent une demande pour des infrastructures, des équipements et des services. Cette demande s'exprime souvent avec un décalage dans le temps qui se situe dans un horizon postérieur au prochain rendez-vous électoral des édiles municipaux. Le cœur du problème réside dans le fait que la réponse à ces nouveaux besoins se trouve à d'autres paliers de gouvernement, ce qui a pour effet de déresponsabiliser les élus locaux. Or, pourquoi les municipalités contrôleraientelles leur développement immobilier quand les gouvernements supérieurs ont jusqu'à maintenant toujours payé pour les infrastructures et les équipements requis?

\section{Si la tendance se maintient : un avenir peu réconfortant}

Ce tour d'horizon porte à croire que le contrôle de l'étalement urbain est impossible et qu'une planifica- 
tion intégrée sur le territoire est utopique. Dans ce portrait, la protection du territoire agricole apparaît précaire et les terres agricoles encore menacées à long terme. Il y a cependant lieu de regarder maintenant la situation en perspective.

Les prévisions démographique du Québec annoncent une stagnation, voire même une diminution de population dans plusieurs régions. Les nouveaux espaces urbanisés répondent davantage à un redéploiement de population plutôt qu'à une croissance. La société québécoise ne peut plus assumer les coûts de l'étalement urbain, alors que les villes-centres sont en déclin au profit de leur périphérie. Aux prises avec une crise permanente des finances publiques, il serait irresponsable de continuer à s'endetter, à gaspiller des ressources financières et naturelles, à tolérer des entraves au développement agricole sur les meilleures terres du Québec. Il faut notamment trouver des moyens d'ajuster la fiscalité municipale de manière à ce que les élus locaux puissent percevoir l'intérêt de l'agriculture pour le développement de leur territoire et reconnaître que cette activité est essentielle à maints égards.

\section{La société québécoise ne peut plus assumer les coûts de l'étalement urbain, alors que les villes-centres sont en déclin au profit de leur périphérie.}

Depuis 25 ans, les énoncés de principes ont semblé à plusieurs reprises vouloir sonner la fin de la récréation et ramener tout le monde à l'ordre et au bon sens, à commencer par l'administration gouvernementale. La pression accrue pour affecter plus de ressources aux soins de santé face une population vieillissante aura-t-elle raison de la concurrence coûteuse et malsaine entre les municipalités périurbaines et entre les villes-centres et leur périphérie ? Dans les faits, plus ça change, plus c'est pareil. Serait-ce à cause d'outils inappropriés ou de la faiblesse de la volonté politique ? Les deux sont un peu vrais, mais il y a surtout un manque flagrant de vision chez les décideurs pour intégrer l'économique, la gestion territoriale de même que les autres dimensions du développement. Pour parodier Shakespeare, on pourrait dire que, décidé- ment, il y a quelque chose qui ne va pas au royaume du Québec.

\section{Notes et références}

1 Union des producteurs agricoles (1998). Pour une politique de développement rural au Québec, mémoire pour la consultation menée par Solidarité rurale, Longueuil, p. 1037.

2 MAPAQ (2002). Le Québec bioalimentraire en un coup d'œil, www.agr.gouv.qc.ca.

3 Binet, Claire (1996). L'étalement urbain et la protection du territoire agricole dans la grande région de Montréal, travail présenté pour le cours «Analyse et gestion de l'environnement » dans le cadre de la maîtrise en Analyse et gestion urbaines (non publié), UQAM; Union des producteurs agricoles (1996). La gestion des matières résiduelles, mémoire présenté au BAPE, Longueuil.

$4 \quad$ Union des producteurs agricoles (1998), op.cit.; Statistique Canada (2001). Recensement agricole, www.statcan.ca.

5 Binet, Claire (1999). Les espaces agricoles des franges urbaines et le contrôle de l'urbanisation: le cas de la rivesud de Montréal, rapport d'activité pour la maitrise en Analyse et gestion urbaines, Montréal, UQAM, p. 67-102.

6 Centre de recherches urbaines et régionales (CRUR-INRS) (1972). Région Sud - L'agriculture, étude interdisciplinaire du territoire de la région sud de Montréal pour l'OPDQ, Montréal; Thibodeau, Jean-Claude (1976). L'impact de Montréal sur les zones agricoles périphériques, Montréal, INRS-Urbanisation.

7 Ministère des Affaires municipales (2001). Cadre d'aménagement métropolitain pour la région de Montréal, p. 4160, www.mamsl.gouv.qc.ca.

8 Binet, Claire (1999), op. cit., p. 103-131.

9 Thibodeau Jean-Claude et al. (1986), Le zonage agricole, un bilan positif, Montréal, INRS-Urbanisation.

10 Duval, Daniel (1990). Développement urbain et zone agricole dans la région de Montréal, mémoire de maîtrise (territoires couverts : CUM et MRC de Laval, Champlain, Lajemmerais et Roussillon), Montréal, Université de Montréal.

11 Ministère des Affaires municipales (2001), op. cit., p. 76.

12 Binet, Claire (1999), op. cit., p. 132-177. 